\title{
Gastric Burkitt's lymphoma in a child: A rare case
}

\author{
ROSSELLA ANGOTTI $^{1}$, MARIO MARINI ${ }^{2}$, GIULIA GIANNOTTI ${ }^{1}$, ANDREINA BURGIO $^{1}$, \\ DANIELA MEUCCI $^{1}$, MARIA PAVONE ${ }^{1}$ and MARIO MESSINA ${ }^{1}$ \\ ${ }^{1}$ Division of Pediatric Surgery, Department of Pediatrics, Obstetrics and Reproductive Medicine, University of Siena; \\ ${ }^{2}$ Department of Pediatric Gastrointestinal Endoscopy A.O.U.S., Policlinico ‘S. Maria alle Scotte', Siena, Italy
}

Received January 27, 2012; Accepted June 15, 2012

DOI: $10.3892 / \mathrm{ol} .2012 .789$

\begin{abstract}
Burkitt's lymphoma (BL) is a high-grade lymphoma which represents $8-10 \%$ of all tumors in individuals younger than 15 years old. It may occur as an abdominal tumor, which in rare cases may include gastric mucosa, although in most cases, the tumor is located in the ileum or cecum. Primary gastric lymphoma constitutes as low as $1.48 \%$ of all gastric cancers in children. In this study, we described a case of gastric $\mathrm{BL}$ in a 4-year-old child, presenting discontinuous abdominal pain, weight loss, constipation and irritability. Despite its rarity in children, this tumor should be treated aggressively and long-term survival has been reported.

\section{Introduction}

Burkitt's lymphoma (BL) is a high-grade mature B-cell neoplasm which represents $8-10 \%$ of all tumors in individuals younger than 15 years old. It is composed of monomorphic medium-size B cells with basophilic cytoplasm and numerous mitotic figures (1-3). BL may occur as an abdominal tumor, which in rare cases may include gastric mucosa (less than $2 \%$ ), although in most cases, the tumor is located in the ileum or cecum. Primary gastric lymphoma constitutes $1.48 \%$ of all gastric cancers in children (4-7). We report the case of a 4-year-old male presenting with discontinuous abdominal pain, weight loss, constipation and irritability with a diagnosis of gastric BL. This study focuses on the significant possibility of BL in children with gastric mass. The patient's family consented to this study.
\end{abstract}

\section{Case report}

A 4-year-old male, with discontinuous abdominal pain, anorexia and weight loss of approximately $3 \mathrm{~kg}$, constipation and irritability, was admitted to our clinic. The patient had presented

Correspondence to: Dr Rossella Angotti, Division of Pediatric Surgery, Department of Pediatrics, Obstetrics and Reproductive Medicine, University of Siena, Policlinico 'S. Maria alle Scotte', Viale Bracci 16, I-53100 Siena, Italy

E-mail: rossellaangotti@libero.it

Key words: gastric Burkitt's lymphoma, children recurrent spasmodic abdominal pain for approximately one month and usually the individual felt better following gas emission. His familial history was negative for lymphoma, gastric carcinoma or other gastrointestinal malignancies. On physical examination the patient presented with pallor and edema of the left eye. There was no respiratory distress, lymphadenopathy, jaundice, abdominal masses or hepatosplenomegaly. The laboratory tests showed the following: anemia (hb $9.8 \mathrm{l} / \mathrm{dl}$ ), elevated white blood cell count (WBC) $\left[13 \times 10^{9}\right.$ cells/1 (of which N 59\%, L 29\%, M 7.6\%, E 3.5\% and B 0.5\%)] and platelet counts $534 \times 10^{9} \mathrm{cells} / 1$. Tests also showed elevated levels of serum lactate dehydrogenase (LDH) $966 \mathrm{U} / 1$ (normal value, 150/500), sedimentation rate $37 \mathrm{~mm} / \mathrm{h}$ and ferritin $5 \mathrm{ng} / \mathrm{ml}$; occult blood in the stool was positive.

The abdominal X-ray with contrast showed an exophitic lesion in the stomach with central ulceration (Fig. 1). A gastroduodenal endoscopy was performed under general anesthesia, which showed an ulcerated masses along the anterior and posterior walls of the gastric body; the remaining gastric wall appeared normal. The morphological features were consistent with the diagnosis of non-epithelial neoplasia (Figs. 2 and 3). Gastric biopsies were performed and histological examination confirmed the diagnosis of gastric BL. Immunohistochemistry revealed that lymphoid cells were positive for CD20, CD10 and CD38 and negative for CD44 and Bcl2. Abdominal, neck, head and testicular ultrasound scans and total body magnetic resonance imaging (MRI) scans were performed. Total body positron emission tomography revealed a large gastric hypermetabolic mass and increased uptake in the bowel, thigh bone and bone marrow. Bone marrow aspirate and cerebrospinal fluid were positive for lymphoma. These examinations showed the presence of diffuse disease. Based on the results of the examinations, the patient was graded as fourth risk group (R4) since LDH levels were greater than 1000 U/1 (1,214 U/1) and bone marrow was involved. The patient was discharged and referred to the Pediatric Oncology Center for chemotherapy.

\section{Discussion}

Non-Hodgkin's lymphoma (NHL) is the third most common cancer $(10 \%)$ and accounts for approximately $60 \%$ of all lymphomas in children and adolescents. The four major pathological subtypes of childhood and adolescents NHL are BL (40\%), lymphoblastic lymphoma (30\%), diffuse large B-cell lymphoma (20\%) and anaplastic large cell lymphoma (10\%) (2). 

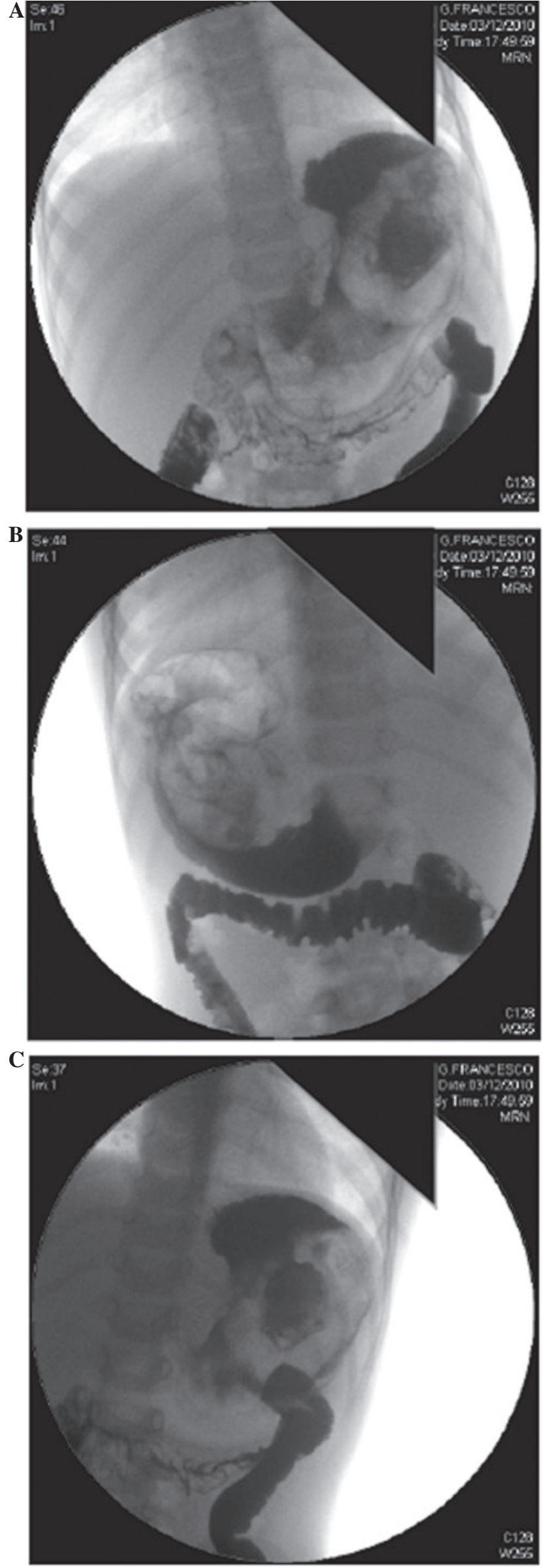

Figure 1. Abdominal X-ray with contrast; exophytic lesion in the stomach with central ulceration was detected.

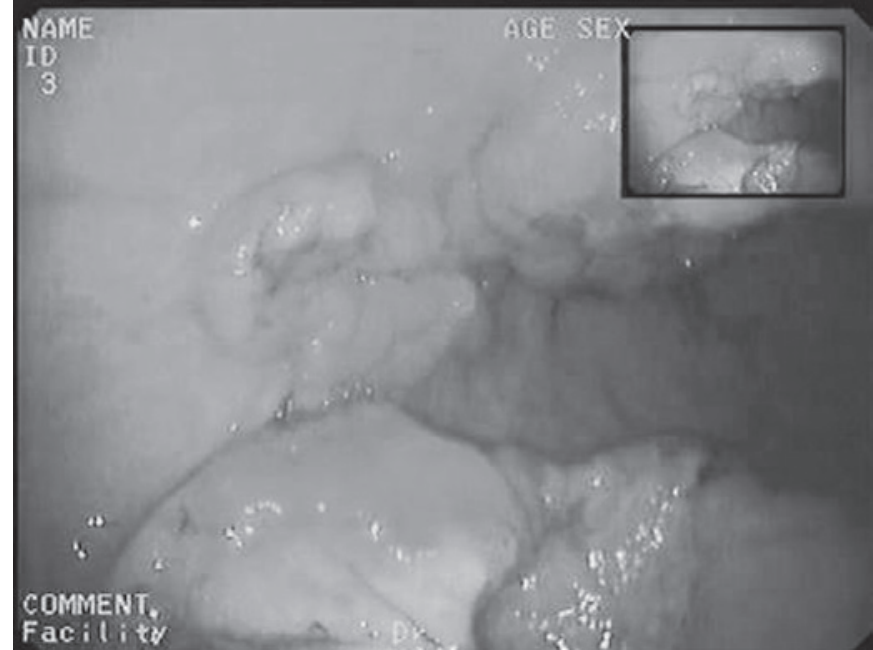

Figure 2. Esophagogastroduodenoscopy (Pentax Endoscope EG340 K) of pseudopolypoid lesions and deep ulceration of the gastric wall.

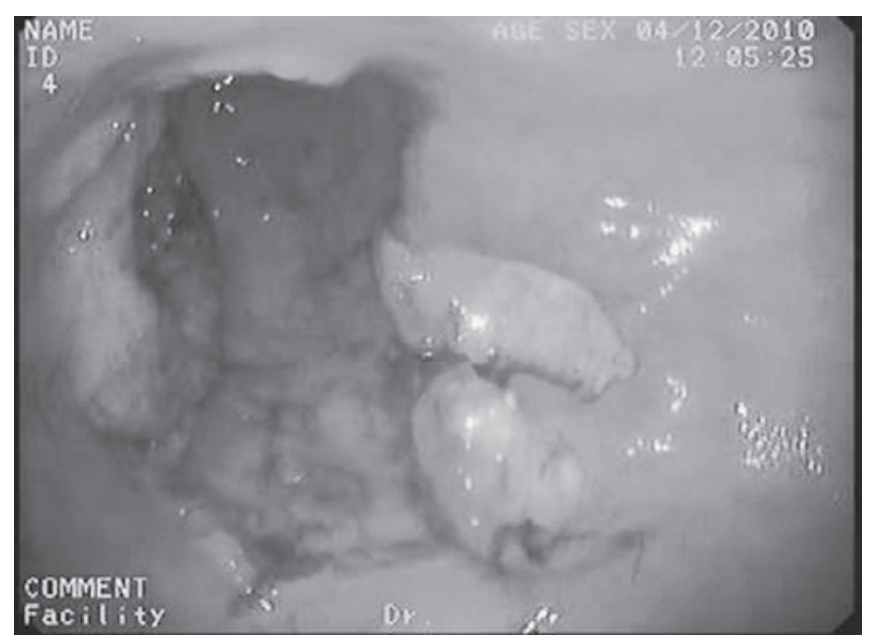

Figure 3. Deep ulceration of the anterior and posterior walls of the gastric body.

Two clinical variants of BL have been recognized by the World Health Organization classification (WHO): endemic and sporadic, which are indistinguishable by histology, but have a different geographical distribution. The etiology of endemic BL is correlated with the Epstein-Barr virus (EBV) and frequently presents with tumors of the head and neck in a population in equatorial Africa. Translocation involving the C-MYC gene is a consistent feature of BL. BL cases have a translocation involving the C-MYC gene at $8 \mathrm{q} 24$ with the immunoglobulin heavy chain gene (IGH) at $14 \mathrm{q} 32$, or, less commonly, with $\kappa$ light chain locus (IGK) at $2 \mathrm{q} 11$ or $\lambda$ light chain locus (IGL) at 22q11 $(8,9)$. The etiology of sporadic BL is unknown; the disease has an abdominal presentation and is the most common type of lymphoma occurring in young children in the USA; the presenting symptoms include: an abdominal mass, intestinal obstruction, intussusceptions and acute abdominal pain. In most cases, the abdominal mass is located in the ileum or cecum, however, in rare cases it can include the gastric mucosa (less than 2\%) (7). Primary gastric lymphoma constitutes as low as $1.48 \%$ of all gastric cancers in 
children (4-6). At present, surgery has a minimal role in the treatment of this pathology since chemotherapy is the mainstay treatment for all variants of BL, due to its extreme sensitivity. A good prognosis has been reported for these patients, however, treatment depends on the stratification of patients into four risk groups (R1-4). R1 includes patients with completely resected disease and a negative histopathological exam of lymph nodes; R2, incompletely resected or unresected disease and extra-abdominal disease localization or abdominal and LDH levels less than $500 \mathrm{IU} / \mathrm{l}$; R3, unresected abdominal lymphoma and LDH levels between 500 and 1,000 IU/1 and patients with bone marrow without CNS (central nervous system) involvement; and R4, LDH levels greater than 1,000 IU/1 and with bone marrow and/or CNS involvement $(10,11)$. The present case shows the importance of considering the possibility of malignancy in children with gastric neoformation or in patients presenting with discontinuous abdominal pain, weight loss and irritability. The literature confirms the rarity of primary gastric lymphoma in children, as less than 20 children have been described during the last decade and most of these lymphomas are high-grade lymphoma $(7,12)$.

\section{References}

1. McLean TW, Farber RS, Lewis ZT, Wofford MM, Pettenati MJ, Pranikoff T and Chauvenet AR: Diagnosis of Burkitt lymphoma in pediatric patients by thoracentesis. Pediatr Blood Cancer 49: 90-92, 2007.

2. Gence A, Sahin C, Celayir AC and Yavuz H: Primary Burkitt lymphoma presenting as a solitary rectal polyp in a child. Pediatr Surg Int 24: 1215-1217, 2008.
3. Bellan C, Lazzi S, De Falco G, Rogena EA and Leoncini L: Burkitt lymphoma versus diffuse large B-cell lymphoma: a practical approach. Hematol Oncol 27: 182-185, 2009.

4. Harris GJ and Laszewski MJ: Pediatric primary gastric lymphoma. South Med J 85: 432-434, 1992.

5. Chieng JH, Garrett J, Ding SL and Sullivan M: Clinical presentation and endoscopic features of primary gastric Burkitt lymphoma in childhood, presenting as a protein-losing enteropathy: a case report. J Med Case Reports 3: 7256, 2009.

6. Kesik V, Safali M, Citak EC, Kismet E and Koseoglu V: Primary gastric Burkitt lymphoma: a rare cause of intraabdominal mass in childhood. Pediatr Surg Int 26: 927-929, 2010.

7. Jacquemart C, Guidi O, Etienne I, Delrez R, Forget P, Dresse MF, Depas G, de Leval L and Hoyoux C: Pediatric gastric lymphoma: a rare entity. J Pediatr Hematol Oncol 30: 984-986, 2008.

8. Mbulaiteye SM, Biggar RJ, Bhatia K, Linet MS and Devesa SS: Sporadic childhood Burkitt lymphoma incidence in the United States during 1992-2005. Pediatr Blood Cancer 53: 366-370, 2009.

9. Cairo MS, Raetz E, Lim MS, Davenport V and Perkins SL: Childhood and adolescent non-Hodgkin lymphoma: new insights in biology and critical challenges for the future. Pediatr Blood Cancer 45: 753-769, 2005.

10. Pillon M, Piglione M, Garaventa A, Conter V, Giuliano M, Arcamone G, Mura R, Cellini M, D'Amore ES, Varotto S, Mussolin L, Rosolen A; AIEOP-NHL Committee: Long-term results of AIEOP LNH-92 protocol for the treatment of pediatric lymphoblastic lymphoma: a report of the Italian Association of Pediatric Hematology and Oncology. Pediatr Blood Cancer 53: 953-959, 2009.

11. Eldar AH, Futerman B, Abrahami G, Attias D, Barak AB, Burstein Y, Dvir R, et al: Burkitt lymphoma in children: the Israeli experience. J Pediatr Hematol Oncol 31: 428-436, 2009.

12. Moschovi M, Menegas D, Stefanaki K, Constantinidou CV and Tzortzatou-Stathopoulou F: Primary gastric Burkitt lymphoma in childhood: associated with Helicobacter pylori? Med Pediatr Oncol 41: 444-447, 2003. 